\title{
Les universités du Québec: situation, orientation, réalisations
}

\author{
Objectifs généraux de l'enseignement supérieur et grandes orientations des établissements. \\ 1973, Conseil des universités.

\section{I - RAPPORT DU COMITÉ DES OBJECTIFS DE L'ENSEIGNEMENT SUPÉRIEUR AU CONSEIL DES UNIVERSITÉS DU QUEBEC}

\author{
René Hurtubise *
}

\begin{abstract}
Résumer en quelques trois pages un tel rapport constitue un défi déraisonnable et injuste... mais telle est la directive.

Le Comité de rédaction formé de quatre personnes, - à savoir : René Hurtubise, directeur général de la Conférence des recteurs et des principaux des universités du Québec, Lorenzo Roy, membre de la Commission de la réforme de l'Université Laval, Pierre W. Bélanger, professeur à la faculté des sciences de l'éducation de l'Université Laval, et André Turmel, chargé de recherche au Conseil des universités, - a présenté la version finale de ce rapport le 7 décembre 1972. En plus de l'introduction qui dénote l'esprit de l'étude, le texte comporte six chapitres portant les titres suivants: 1 . les universités dans un monde en changement ; 2 . l'éducation permanente, facteur intégrateur de toute politique d'éducation ; 3 . l'enseignement collégial et le premier cycle universitaire ; 4. les études avancées et la recherche; 5 . le milieu universitaire québécois, et 6 . l'organisation du système universitaire québécois.
\end{abstract}

L'introduction rappelle certaines contraintes que tout lecteur doit avoir à l'esprit, e.g. contrainte espace en ce que la réflexion est prioritairement axée sur l'université québécoise, contrainte temps en ce que cette réflexion se limite à la prochaine décennie. Et les auteurs situent leur apport dans les termes suivants : «Les lecteurs voudront

* René Hurtubise, directeur général de la Conférence des recteurs el des principaux des universités du Québec. 
bien voir dans ce projet une contribution des auteurs au débat actuellement en cours dans les sociétés occidentales sur la crise de l'université et la crise du savoir. »

Dans le chapitre 1 , après avoir constaté une situation d'urgence résultant d'un sous-développement relatif sur les plans économique, social et culturel par rapport au Canada et aux États-Unis, l'on soumet que le cœur du problème de l'université en est un de définition des objectifs. Ce qui amène à distinguer les objectifs généraux, qui sont trans-culturels, des objectifs spécifiques, lesquels varient d'un pays à l'autre et même d'une institution à l'autre. Il est alors proposé «que l'objectif majeur de l'enseignement universitaire québécois durant la prochaine décennie soit d'apporter une contribution significative et qualitative au développement économique, social et culturel au Québec», étant entendu que le but ultime du développement est le souci de la qualité de la vie.

Le chapitre 2 rappelle qu'à la base du renouvellement se trouve le droit fondamental de tout individu à l'auto-actualisation mais il s'agit bien sûr d'un individu en situation, lui-même membre d'un groupe social en situation. Ce qui amène à conclure que toute éducation doit être «pensée et vécue comme étant permanente, continue et récurrente ». C'est ainsi que l'éducation permanente devient le facteur intégrateur de toute politique d'éducation, y compris la politique universitaire. Suit alors l'énumération des principales règles du jeu: accessibilité universelle et égalité d'éducabilité, ouverture, souplesse et responsabilité devant les changements de demande, pluralisme et diversité, rationalisation dans l'utilisation des ressources, diversité des approches pédagogiques et des modes d'apprentissage et enfin, dans le contexte d'une véritable éducation continue, réflexion en profondeur et remise en cause de la structure et de la durée des études dans l'approche d'un premier cycle plus polyvalent basé sur des prémisses spécifiées... Et ce chapitre se termine par la distinction entre éducation permanente et éducation des adultes et y va d'un certain nombre de suggestions concrètes.

Le chapitre 3, après avoir déploré une certaine spécialisation au niveau des profils d'admission et un cloisonnement trop rigide entre le Cegep et l'université, s'attarde à définir les objectifs du 1er cycle de l'enseignement universitaire et prône une réorientation des curriculum distinguant, comme c'est inévitable, entre les curriculum disciplinaires, professionnels et ceux axés sur des champs d'études non-professionnalisés, insistant sur le fait qu'il ne s'agit pas là d'un exercice désincarné et mentionnant que l'on est déjà à l'œuvre aux États-Unis dans ce domaine.

Le chapitre 4 est consacré aux études avancées et à la recherche et souligne un état de sous-développement relatif par rapport à la situation de l'ensemble du Canada. Il se termine par la suggestion de créer un centre de synthèse interdisciplinaire qui «vise d'abord à trouver et à assurer les fondements scientifiques de l'interdisciplinarité par l'entremise de la recherche », quitte à prévoir subséquemment des retombées sur l'enseignement.

Le chapitre 5 aborde le délicat et complexe problème du milieu universitaire québécois, à savoir les étudiants, les professeurs, les chercheurs et les administrateurs. 
Au sujet des étudiants, il amorce timidement une esquisse de solution : des itinéraires variés. Concernant les professeurs et les chercheurs, il insiste d'abord sur les défis multiples et écrasants auxquels ils font face et émet l'opinion que l'avenir repose sur une " conception évolutive du rôle du professeur et du chercheur qui résiste à une cristallisation hâtive de ses responsabilités conçues davantage face au passé plutôt qu'à l'avenir ». Puis le rapport consacre quelques pages au statut des professeurs, à leurs revendications et aux formules diverses de regroupement. Enfin, face aux administrateurs, l'étude souligne l'existence d'un nouveau style de gestion, le développement de l'appareil administratif et la nécessité d'une préparation adéquate en mentionnant l'utilité d'un bureau d'études et d'analyses comme auxiliaire de l'administration.

Le dernier chapitre, tel que déjà mentionné, est consacré à l'organisation du système universitaire québécois. Passant rapidement sur la situation actuelle et la théorie des mécanismes de coordination, il s'arrête plus longuement à certains axes de réflexion tels les rôles des éléments du système et les modalités de réalisation.

En conclusion, après avoir affirmé qu'ils sont conscients des limites de leur travail, les auteurs terminent par ces deux paragraphes:

«Nous avons voulu concevoir des objectifs de l'enseignement supérieur au Québec et les intégrer dans un système où le jeu des tensions produirait un dynamisme. Les objectifs globaux que nous proposons sont bipolaires et en tension. On retrouve des tensions à l'intérieur de tous les autres éléments du système: intégration des adultes avec les jeunes; formation polyvalente vs formation spécialisée; satisfaction des besoins sociaux, épanouissement de la personne et maintien de la qualité ; recherche fondamentale et recherche appliquée; centralisation au niveau provincial et autonomie des institutions $》$.

«Les tensions sont présentes partout et elles devront être administrées de façon que leur présence ne rompe pas le mouvement de l'équilibre du système. Mais elles devront persister car elles sont la condition d'un système dynamique dans la société moderne, système qui puisse s'adapter aux changements sociaux, répondre aux nouveaux besoins sans être exclusivement à leur remorque ».

\section{II - OBJECTIFS DE L'ENSEIGNEMENT SUPERIEUR ET GRANDES ORIENTA- TIONS DES ETABLISSEMENTS}

\section{Michel Leclerc*}

Le troisième cahier du document Objectifs de l'enseignement supérieur et grandes orientations des établissements du Conseil des Universités du Québec traite des orientations de l'enseignement supérieur dans les années '70; ce cahier de 259 pages (dans

* Michel Leclerc, directeur de la recherche et de la planification, Conférence des recteurs des universités du Québec. 
sa version deuxième et définitive) comprend une introduction et trois parties : la première sur l'évolution de l'enseignement supérieur durant la décennie 1970, la deuxième sur le fonctionnement du résean universitaire et la troisième sur les orientations de chaque établissement.

Le cahier troisième, avec sa table des matières en cinq pages, et ses dix-huit sections de recommandations se laisse difficilement résumer en une page : il convient, cependant, de souligner qu'il constitue un effort remarquable dans la voie d'une orientation à moyen terme des universités du Québec puisqu'il dégage les axes de développement de chacune des universités (dans la 3e partie) permettant ainsi de mieux cerner les zones de coopération possible entre chaque établissement.

De la première partie du cahier, nous retiendrons deux types de prévisions: les projections des étudiants à temps complet par institution jusqu'en 1976-77 et les prévisions des dépenses et subventions de fonctionnement universitaire prévues pour 1976.

Après avoir souligné l'écart important entre les prévisions démographiques des universités et celles du ministère de l'éducation, après avoir rappelé «qu'il est difficile de présenter à l'heure actuelle des prévisions quelque peu fermes» (p. 10) le conseil souligne l'augmentation rapide des inscriptions à temps partiel, et affirme «que les uni-

\begin{tabular}{|c|c|c|c|c|c|}
\hline & 1971.72 & $1972-73$ & $1973-74$ & 1974.75 & $1975-76$ \\
\hline \multicolumn{6}{|c|}{ Total Québec (y compris « Recherche» provinciale) } \\
\hline Hypotbèse faible & & & & & \\
\hline Subv, de recherche $(*)$ & 213,300 & 1,000 & 2,000 & 3,000 & 4,000 \\
\hline Revenus totaux $(\$ 000)$ & 177,100 & 244,900 & 269,100 & 293,600 & 323,900 \\
\hline Subv, totales $(\$ 000)$ & & 205,600 & 228,600 & 250,300 & 280,800 \\
\hline Augment. des revenus (\%) & & 14.9 & 9.9 & 9.1 & 10,3 \\
\hline Auginent. des subvent. $(\%)$ & & 16.1 & 11.2 & 9.5 & 12.2 \\
\hline Hypotbèse forte & & & & & \\
\hline $\begin{array}{l}\text { Subv, de recherche }(*) \\
(\$ 000)\end{array}$ & & 2,000 & 4,000 & 6,000 & 8,000 \\
\hline Revenus totaux $(\$ 000)$ & 213,300 & 253,700 & 288,500 & 323,500 & 368,300 \\
\hline Subv. totales $(\$ 000)$ & 177,100 & 214,400 & 248,000 & 281,400 & 325,200 \\
\hline Augment. des revenus $(\%)$ & & 18.9 & 13.7 & 12.1 & 13.8 \\
\hline Augment. des subvent. (\%) & & 21.0 & 15.6 & 13.5 & 15.2 \\
\hline
\end{tabular}

(*) Au-delà des subventions accordées en 1971-72. 
versités anglophones ne connaîtront aucun développement au cours des cinq prochaines années » et que «les universités francophones verront en gros leurs effectifs s'accroître dans les cadres physiques actuels (à l'exception de l'Université du Québec)» (p. 11). Plus précisément, les universités recevraient 64,500 étudiants à temps complet en 1976-77, ce qui constituerait une augmentation annuelle d'environ 1.5\% de 1971-72 à 1972-77 : les universités anglophones, «d'où auront disparu les étudiants de niveau collégial, n'accueilleront plus en 1976-77, qu'environ 18,000 étudiants à temps complet, contre plus de 20,000 en 1971-72 », (p. 11) soit une diminution de $11 \%$. Les universités francophones verraient leurs effectifs passer de 39,000 à 47,000 étudiants à temps complet, soit une augmentation annuelle d'environ $3 \%$.

Au chapitre des dépenses et subventions de fonctionnement, le conseil, à l'aide de cinq paramètres ( $\mathrm{p} .33$ ), établit deux hypothèses, l'une forte et l'autre faible : nous reproduisons une partie (le total) des prévisions du conseil (p. 36).

La deuxième partie, composée de quatre chapitres, traite des caractéristiques générales du réseau, des fonctions communes des universités, de la répartition des fonctions spécifiques des établissements et de certains cas particuliers (les conservatoires, le devenir de l'institut national de la recherche scientifique).

La troisième partie qui traite des orientations spécifiques de chaque établissement délimite les axes de développement et les secteurs modèles d'activités pour chaque université : cette partie constitue d'ailleurs la suite logique du premier cahier qui présentait les caractéristiques de l'enseignement supérieur pour les dix dernières années et se terminait par les orientations que les institutions souhaitaient se donner. 